\title{
An Original Method to Quantify Mitochondria Movement in Cultured Cardiomyocytes
}

\author{
S Pelloux ${ }^{1,3}$, C Ojeda $^{2}$, Y Tourneur ${ }^{1,3}$ \\ ${ }^{1}$ Inserm EMIU 0226, Claude Bernard University, Lyon, France \\ ${ }^{2}$ Inserm ERIT-M 107, Bron, France \\ ${ }^{3}$ Centre Commun de Quantimétrie, Claude Bernard University, Lyon, France
}

\begin{abstract}
We studied in confocal microscopy non-beating cells from the HL-1 cardiac cell line. Using a fluorescent dye, mitochondria appeared as a dense tubular network, in permanent fusion, fission and displacement. To quantify these movements in response to pharmacological stimuli, we needed a method only based on their position.

Time-lapse recording was performed in physiological medium. Every image was first converted into binary images by ultimate erosion or skeletonization. Then a distance map was created by attributing to every point a grey level equal to the distance from the skeleton. In a third step, the following binary image was laid over the distance image, by pixel to pixel multiplication. The pixel value below the binary objects indicated the distance ran between the two images.

The method is fast and accurate, and can be scaled in absolute values. The average velocity lies in the range obtained by other methods.
\end{abstract}

\section{Introduction}

Confocal microscopy allows the observation of living cells. Mitochondria can be stained using fluorescent

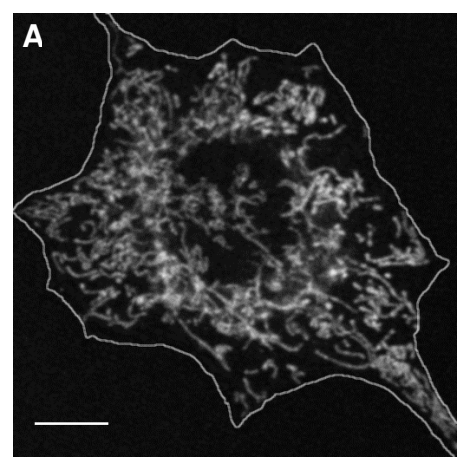

cationic dyes, which accumulates inside the matrix following the electrical gradient. Video microscopy reveals cardiac mitochondria as a dense tubular network. Their intensity and apparent diameter change with time.

To quantify mitochondria movements in response to pharmacological stimuli, we needed a method only based on their position. Classical tracking methods are inappropriate to follow movements of fusion and fission. We developed a stable method to estimate mitochondrial movement based on the image analysis tool of distance map.

\section{Methods}

We studied in confocal microscopy non-beating cells [1] from the first cardiac cell line, HL-1, kindly provided by $\mathrm{Dr}$ W. Claycomb [2]. Cells were incubated with a mitochondrial selective fluorescent dye, a rhodamine derivative, TMRM (Molecular Probes, Eugene, OR) for $15 \mathrm{~min}$ before the experiment.

Time-lapse recording was performed using a $63 \mathrm{X}$ (N.A. 0.9) water immersion objective in physiological medium, and one image Ii, (i=1...n) was taken every 15 seconds (Figure 1). The time-series images were analyzed using the imageJ software (NIH, Bethesda, MD).

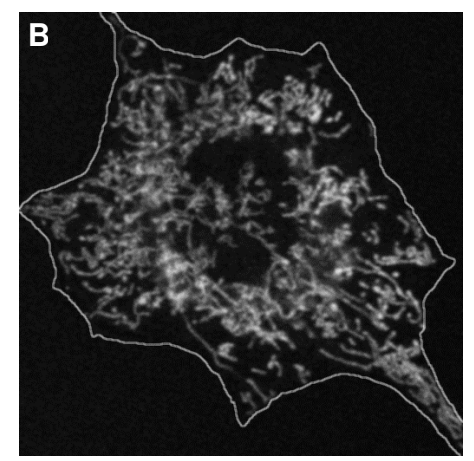

Figure 1. A and B are two images of the same cell (edges in white) stained with TMRM (in grey). A: $t=0 s, B: t=15 s$. Time-lapse observations show that mitochondrial fluorescence change in intensity and in position. Scale bar: $10 \mu \mathrm{m}$. 


\section{Results}

\subsection{Transformation into binary objects}

The first step of the analysis consisted in transforming every grey level image Ii into a binary image (Bi), to be independent of mitochondria intensity and width.

Two different methods were used: the brightest points were extracted by ultimate erosion (Figure $2 \mathrm{C}$, D), the mitochondrial network was represented by a binary skeleton (Figure 2 E, F).We developed a general method to quantify either the dots or the skeleton movement.
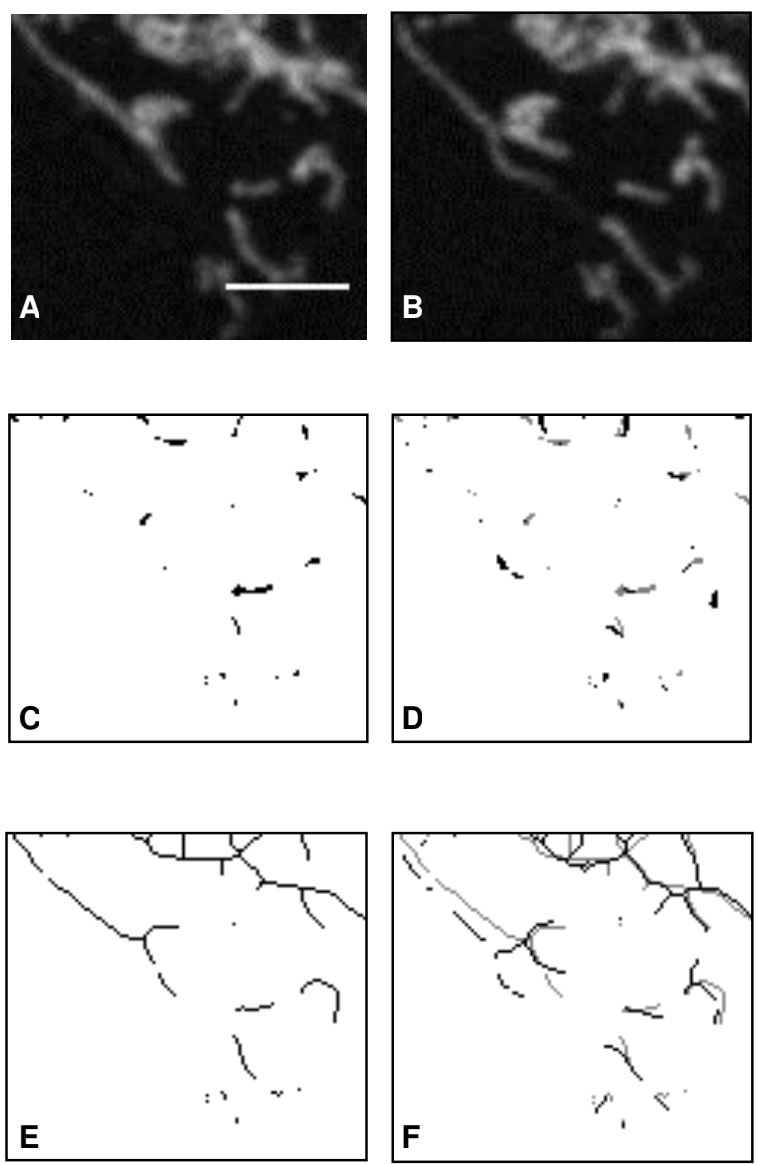

Figure 2. A detail from Figure $1 \mathrm{~A}$ and $\mathrm{B}$ was transformed into binary images. C and D: after ultimate erosion, $\mathrm{E}$ and $\mathrm{F}$ : after skeletonization. Left column: $\mathrm{t}=0 \mathrm{~s}$. Right column: $\mathrm{t}=15 \mathrm{~s}$. In D and $\mathrm{F}$ : superimposition of the preceding image in grey. Scale bar: $5 \mu \mathrm{m}$.

\subsection{Creation of distance maps}

In a second step, a distance map Di was computed for every $\mathrm{Bi}$ in a time-series. From a binary image, the distance map represents at any point in a plane the distance to the nearest object.

This method was applied either to the dots (Figure 3 A) or to the skeleton (Figure $3 \mathrm{~B}$ ). The distance map can be represented in 3D (Figure 3) or in false colors. Such image is black (zero) for points located on the binary object and brighter for more distant points.

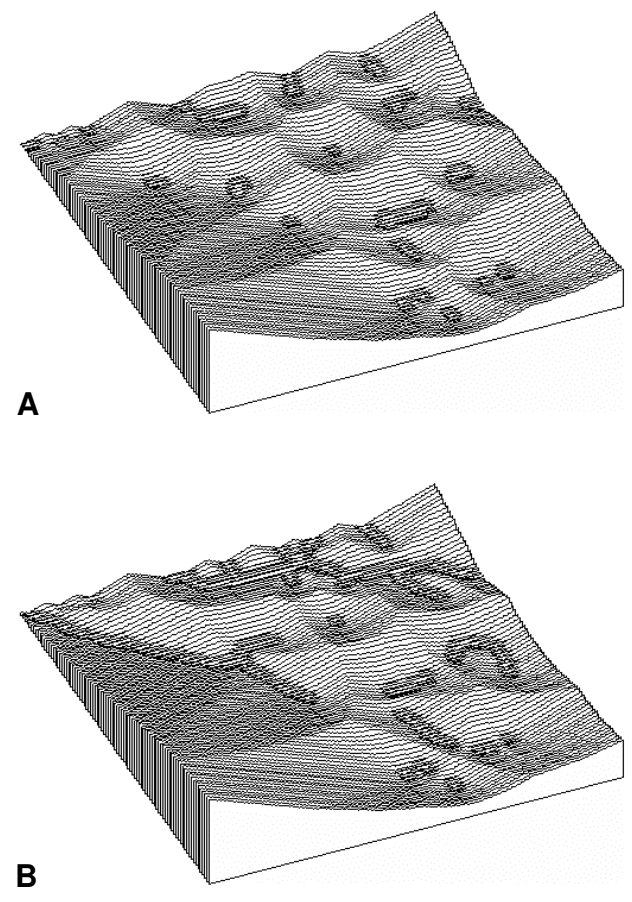

Figure 3. Same detail as in Figure 2 A, transformed into binary images, was converted into distance maps (here, three-dimensional representation). A: after ultimate erosion, B: after skeletonization.

\subsection{Estimation of the displacement}

In a third step, the binary image at time $\mathrm{i}+1, \mathrm{Bi}+1$, was laid over the distance image $\mathrm{Di}$, by pixel to pixel multiplication. 
In the absence of movement, the pixels on the distance map below the dots/skeleton are black. If a movement occurred between the two images, the pixel value below the dots/skeleton indicates the distance ran between times $i$ and $i+1$.

The average value of the multiplication quantifies the average displacement between two successive images (see Figure 4).
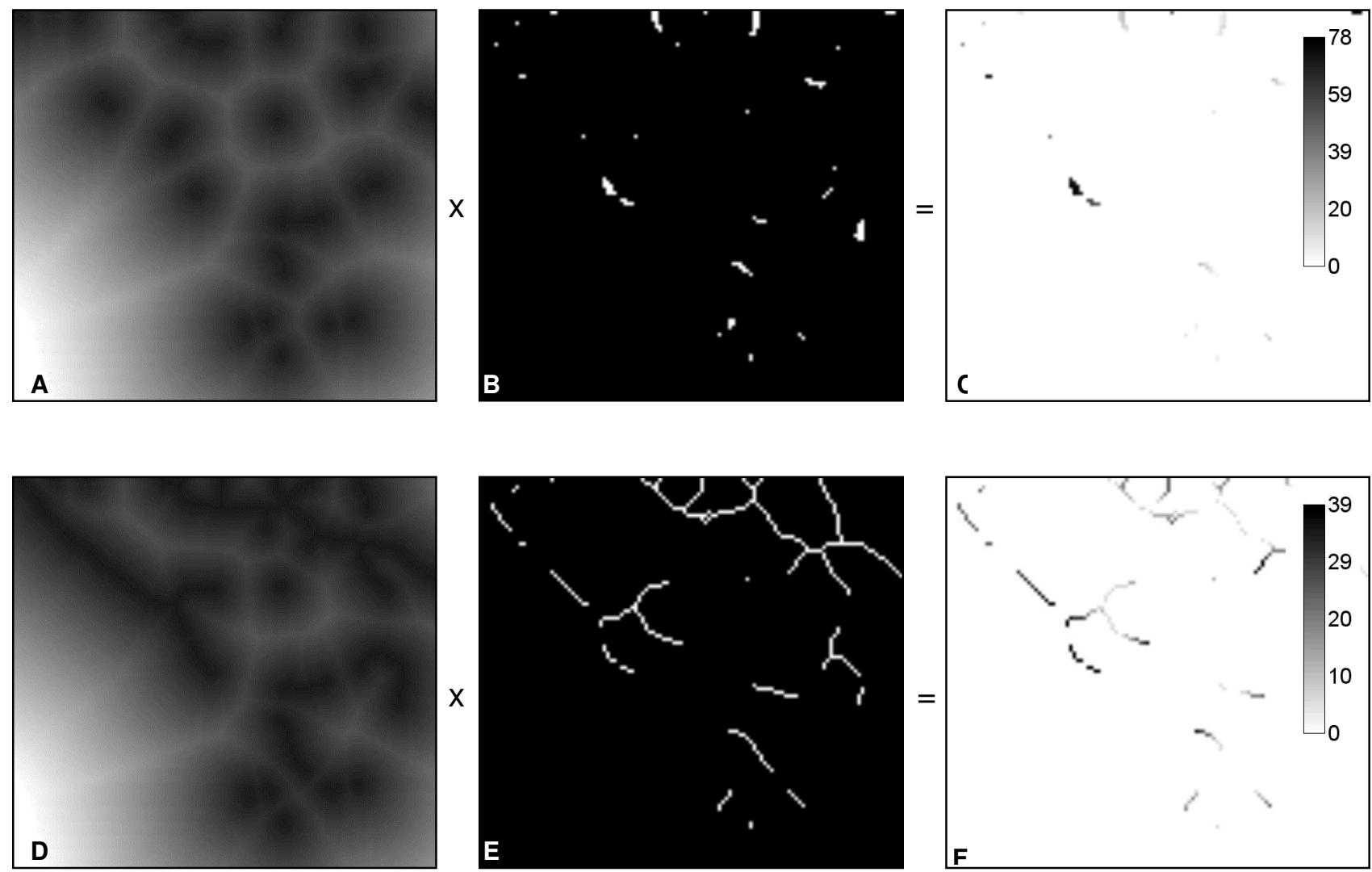

Figure 4. An estimation of the displacement of the binary objects was obtained by multiplying each distance map Di with the successive binary image $\mathrm{Bi}+1$. A - C: after ultimate erosion, D - F: after skeletonization. A, D: distance maps Di. B, $\mathrm{E}$ : binary images $\mathrm{Bi}+1$. $\mathrm{C}, \mathrm{F}$ : result of the multiplication of $\mathrm{Di}$ and $\mathrm{Bi}+1$, the grey level of each pixel represents the movement calibrated in $\mathrm{nm} / \mathrm{s}$.

\section{Discussion and conclusions}

In cultured cells, mitochondria undertake constant movements. In confocal microscopy they appear as a complex network with brighter dots. We developed a method to quantify their average movement. This method could be applied either to the dots or to the global network.
The method is fast and accurate, and can be scaled in absolute values. The results (Figure 5) obtained lie in the range obtained by other authors with different techniques. It can be used as a tool to test pharmacological effects on mitochondria. 


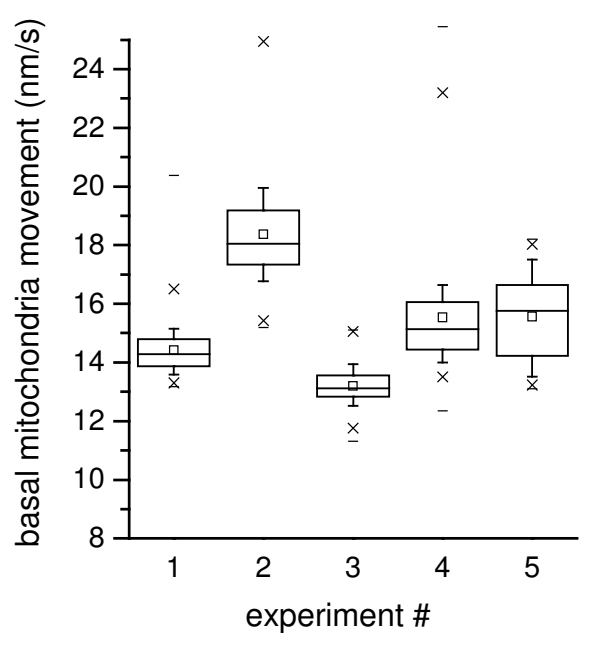

Figure 5. By skeletonization, the average velocity from independent experiments was found to be $15 \mathrm{~nm} / \mathrm{s}$. Ultimate erosion gave a significantly higher value of 33 $\mathrm{nm} / \mathrm{s}$. Both were in the range found in other preparations: $23 \pm 11$ to $49 \pm 21 \mathrm{~nm} / \mathrm{s}$ [3]. The difference between the dots velocity and the skeleton velocity may reflect faster mitochondria (dots) movement within the moving mitochondrial network.

\section{Acknowledgements}

The authors are thankful to Dr W. Claycomb for his kind gift of HL-1 cells.

\section{References}

[1] Claycomb WC, Lanson NA, Jr., Stallworth BS, Egeland DB, Delcarpio JB, Bahinski A, et al. HL-1 cells: a cardiac muscle cell line that contracts and retains phenotypic characteristics of the adult cardiomyocyte. Proc Natl Acad Sci U S A 1998;95(6):2979-84.

[2] Pelloux S, Robillard J, Ferrera R, Bilbaut A, Ojeda C, Saks V, et al. Non-beating HL-1 cells for confocal microscopy: Application to mitochondrial functions during cardiac preconditioning. Prog Biophys Mol Biol 2005 (in press).

[3] Simon VR, Swayne TC, Pon LA. Actin-dependent mitochondrial motility in mitotic yeast and cell-free systems: identification of a motor activity on the mitochondrial surface. J Cell Biol 1995;130(2):345-54.

Address for correspondence

$\begin{array}{ll}\text { Name } & \text { Yves Tourneur } \\ \text { Full postal address } & \begin{array}{l}\text { Centre Commun de Quantimétrie } \\ \text { Université Claude Bernard Lyon1 } \\ \text { 8 Avenue Rockefeller }\end{array} \\ & \text { 69373 Lyon Cedex 08, France } \\ \text { E-mail address } & \text { yves.tourneur@univ-lyon1.fr }\end{array}$

